\title{
Acquired elastotic hemangioma, a little-known entity: report of a case with 15 years of evolution*
}

\author{
Maria Claudia Alves Luceㄹ, Caroline Paias Ribeiro ${ }^{1}$, Bethânia Cabral Cavalli Swiczar¹, \\ Neusa Yuriko Sakai Valente ${ }^{1,2}$
}

DOI: http:/ / dx.doi.org/10.1590/abd1806-4841.20187376

\begin{abstract}
Acquired elastotic hemangioma is a hemangioma variant first described in 2002. It is characterized by being a benign, solitary, slow-growing lesion, that appears in adulthood and is associated with solar exposure. It is a rare hemangioma variant with few cases reported in the literature. We present a case of acquired elastotic hemangioma on the back of the right hand and forearm in a male adult. Acquired elastotic hemangioma is a benign vascular proliferation associated with solar exposure, usually assymptomatic. It affects adults between 59 and 65 years of age. Histopatologically it is characterized by proliferation of small vessels in the upper dermis that are disposed parallel to the epidermis, and significant solar elastosis. The treatment is surgical, with no relapses reported.
\end{abstract}

Keywords: Hemangioma; Neoplasms, vascular tissue; Solar radiation; Vascular neoplasms

\section{INTRODUCTION}

Acquired elastotic hemangioma (AEH) is a hemangioma variant, first described by Requena et al. in 2002. ${ }^{1}$ It is characterized by a solitary, slow-growing lesion located in a sun-exposed area. ${ }^{1,2}$ Histopathologically, it presents a proliferation of capillaries in the superficial dermis, accompanied by a pronounced solar elastosis. ${ }^{1}$ We report a case of this rare hemangioma variant, with characteristic clinical and histopathological aspects.

\section{CASE REPORT}

A 65-year-old man presented with a lesion on the back of his right hand and forearm. The lesion had persisted for 15 years and was asymptomatic and slow growing. Clinical examination revealed a slightly-raised, well-defined erythematous lesion of irregular shape, measuring $8 \mathrm{~cm}$ in the long axis, located on the back of the right hand and forearm, with fading under diascopy. (Figure 1).

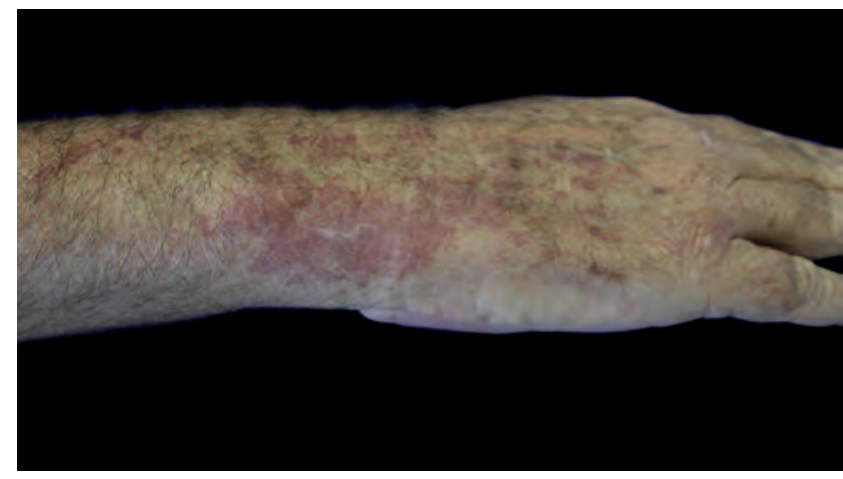

FIGURE 1: Slightly raised erythematous lesion, with irregular border, measuring about $8 \mathrm{~cm}$ in diameter

\footnotetext{
Received 17 August 2017.

Accepted 20 November 2017.

* Work conducted at the Hospital do Servidor Público Estadual de São Paulo, São Paulo (SP), Brazil.

Financial support: None.

Conflict of interests: None.

Dermatology Service, Hospital do Servidor Público Estadual de São Paulo, São Paulo (SP), Brazil.

Dermatopathology Service, Hospital do Servidor Público Estadual de São Paulo, São Paulo (SP), Brazil.
}

MAILING ADDRESS:

Maria Claudia Alves Luce

E-mail: mcluce@gmail.com

(C)2018 by Anais Brasileiros de Dermatologia 
Dermatoscopy showed punctiform and glomerular vessels in homogeneous arrangement (Figure 2). An incisional biopsy was performed. The histopathological examination revealed a proliferation of small, venule-like vessels in the upper dermis, distributed parallel to the epidermis, amid intense solar elastosis (Figures 3 and 4).

\section{DISCUSSION}

$\mathrm{AEH}$ is a benign vascular proliferation, first described in 2002 by Requena et al. ${ }^{1}$ It is a cutaneous hemangioma variant associated with sun exposure. Typically, it presents as a flat or raised, erythematous or violaceous, irregular, slow-growing, solitary plaque. ${ }^{1-4}$

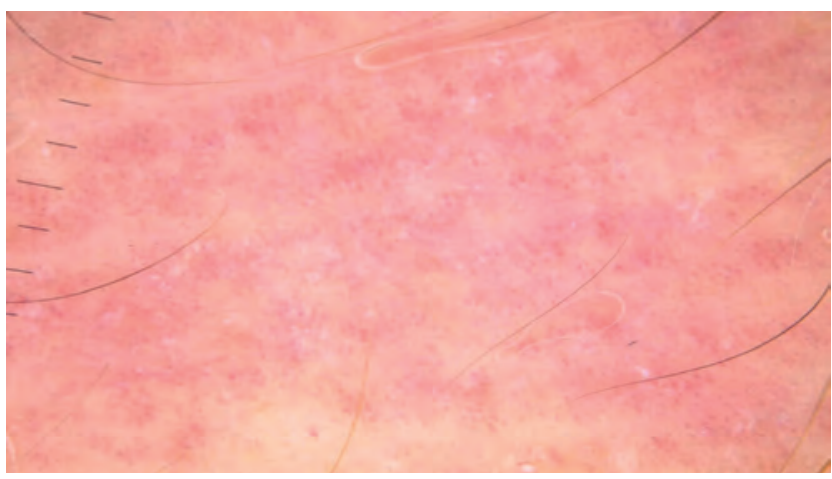

FIGURE 2: Punctiform and glomerular vessels distributed diffusely and homogeneously on dermatoscopy

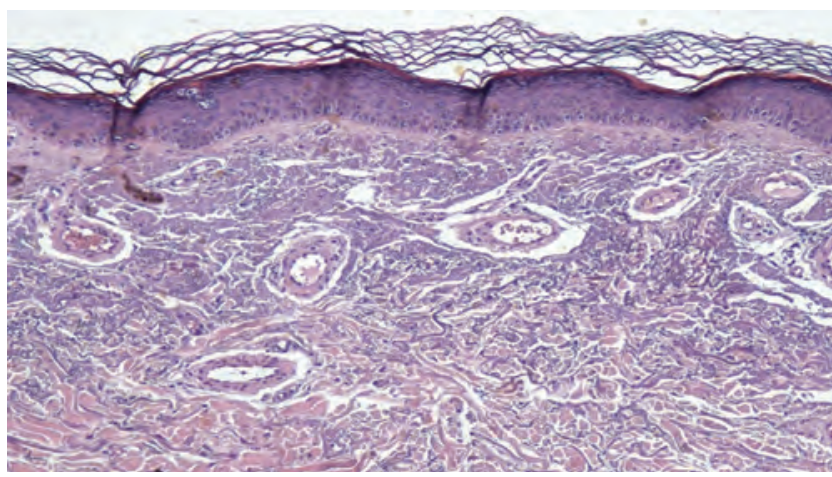

FIGURE 3: Vessel proliferation in the upper dermis and solar elastosis (Hematoxylin \& eosin, x100)

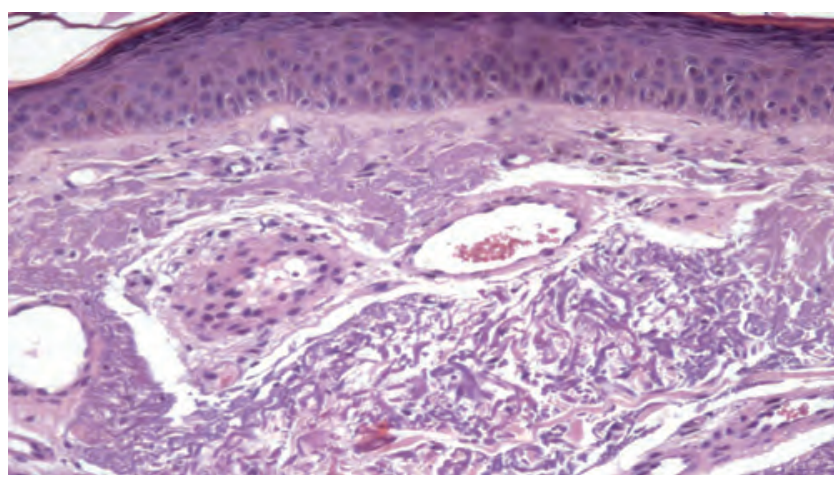

Figure 4: Vessels arranged parallel to the epidermis (Hematoxylin \& eosin, $x 200)$
AEH is usually asymptomatic, and rarely painful. . It is located in areas exposed to sun, particularly the forearms and the neck. ${ }^{1,2}$ Its first description reported cases in women only; however, it has been found in similar proportions of both sexes..$^{1-3}$ It affects mainly adults between 59 and 64 years of age. ${ }^{1,2}$

Our case is similar to previously reported cases in that the patient is an adult and has a single, slow-growing lesion in an area susceptible to sun damage. However, unlike other published cases, our patient's lesion showed a more irregular border, larger dimensions, and prolonged evolution. In the previously described cases, the lesion is about 2 to $5 \mathrm{~cm}$ in diameter, in contrast to $8 \mathrm{~cm}$ in our case (Figure 1). One hypothesis for this difference in that the long evolution period (15 years). Histopathology revealed the same characteristics as in other cases, confirming the diagnosis of AEH.

Diagnosis is based on histopathological features, with vascular proliferation in the superficial dermis, with the vessels forming a band arranged horizontally and parallel to the epidermis. The first description [Requena et al. ${ }^{1}$ highlighted six cases with proliferation of capillaries involving the superficial dermis. In one of these cases, vessels with thickened walls were found, and another patient had proliferation of capillaries and venules. The vast majority of subsequent cases described only capillaries involved in characteristic vascular proliferation. In the case described here, venule-like vessels and rare capillaries were seen in the superficial dermis. There were no endothelial cells with atypia or mitotic figures, an important indication of the benign nature of the affection, distinguishing it from angiosarcoma or Kaposi's sarcoma. There was intense solar elastosis involving the proliferated vessels, suggestive of sun damage as an etiological factor. ${ }^{1}$ In a series of 14 cases, the immunohistochemical studies were all positive for D2-40. In another series with 10 patients, however, only one was positive for D2-40, leaving open the actual participation of lymphatic vessels in AEH. ${ }^{2,3}$

All cases reported in the literature, including the one presented here, were biopsied, with diagnostic hypotheses other than $\mathrm{AEH}$. Among the considered hypotheses, the main one was basal cell carcinoma. Other clinical differential diagnoses are Bowen's disease, actinic keratosis, and other hemangiomas. ${ }^{1-5}$ The histopathological differential diagnosis involves vascular lesions that develop in adulthood, such as senile angioma (a more common, acquired, vascular proliferation of small, dilated, and congested vessels in the dermis, not arranged parallel to the epidermis, with peripheral epithelial collarette ); tufted angioma (multiple lobes composed of endothelial cells and small vessels in the dermis and hypodermis, and a "cannonball" appearance); and the initial stage of Kaposi's sarcoma (presence of predominantly lymphoplasmacytic inflammatory infiltrate, with irregular vascular proliferation dissecting the dermal collagen, and mild atypia of endothelial cells). ${ }^{1,2,4,5}$

The treatment of AEH is surgical, without reports of recurrence. Due to the extent of the lesion in the presented case, clinical follow-up was chosen.

$\mathrm{AEH}$ is probably underdiagnosed, often interpreted as conventional hemangioma. ${ }^{5}$ Thus, we highlight the importance of understanding this new hemangioma variant for generating diagnostic hypotheses on acquired vascular lesions in adults. 


\section{REFERENCES}

1. Requena L, Kutzner $\mathrm{H}$, Mentzel T. Acquired elas- totic hemangioma: a clinicopathologic variant of hemangioma. J Am Acad Dermatol. 2002;47:371-6.

2. Martorell-Calatayud A, Balmer N, Sanmartín 0, Díaz-Recuero JL, Sangueza OP. Definition of the features of acquired elastotic hemangioma reporting the clinical and histopathological characteristics of 14 patients. J Cutan Pathol. 2010;37:460-4.

3. Tong PL, Beer TW. Acquired elastotic hemangioma: ten cases with immunohistochemistry refuting a lymphatic origin in most lesions. J Cutan Pathol. 2010;37:1259-60.
4. Sánchez GF, Calb IL. Hemangioma elastótico adquirido. Dermatol Argent. 2013;19:305-7.

5. García ES, Torres AM, Enríquez BR, Vizcaíno JSS, Gabaldón VH. Hemangioma elastótico adquirido ¿Una entidad infradiagnosticada? Rev Esp Patol. 2004;37: 329-31.

\section{AUTHORS CONTRIBUTION}

Maria Claudia Alves Luce

Conception and planning if the study; Elaboration and writing of the manuscript; $\mathrm{Ob}$ taining, analyzing and interpreting the data; Intellectual participation in propaedeutic and/or therapeutic conduct of cases studied

Caroline Paias Ribeiro

D ORCID 0000-0002-1226-3487

Conception and planning if the study; Intellectual participation in propaedeutic and/or therapeutic conduct of cases studied

\section{Bethânia Cabral Cavalli Swiczar \\ ORCID 0000-0003-2201-5628}

Approval of the final version of the manuscript; Effective participation in research orientation; Intellectual participation in propaedeutic and/or therapeutic conduct of cases studied; Critical review of the literature; Critical review of the manuscript

Neusa Yuriko Sakai Valente

ORCID 0000-0002-8065-2695

Approval of the final version of the manuscript; Effective participation in research orientation; Intellectual participation in propaedeutic and/or therapeutic conduct of cases studied; Critical review of the literature; Critical review of the manuscript

How to cite this article: Luce MCA, Ribeiro CP, Swiczar BCC, Valente NYS. Acquired elastotic hemangioma, a little-known entity: report of a case with 15 years of evolution. An Bras Dermatol. 2018;93(4):559-61 